\title{
Crystalline Silicates in Space
}

\author{
L.B.F.M. Waters \\ Astronomical Institute, University of Amsterdam, Kruislaan 403, \\ NL-1098 SJ Amsterdam, The Netherlands
}

\section{F.J. Molster}

School of Materials Science and Engineering, Georgia Tech, Atlanta, GA 30332-0245, U.S.A. and MVA Inc, Norcross, GA 30093, U.S.A.

\begin{abstract}
An overview is given of the occurrence and properties of crystalline silicates in space. Crystalline silicates are found in circumstellar shells of both young and evolved stars, and are present in Solar System comets. These silicates are Mg-rich and Fe-poor, in contrast to amorphous silicates. We discuss the formation mechanism of crystalline silicates and the apparent lack of these grains in the interstellar medium.
\end{abstract}

\section{Properties and occurrence of crystalline silicates}

Silicates are ubiquitous in interstellar space and are found around both young and evolved stars. Until recently, observations of the thermal infrared emission of silicates showed the presence of broad bands at about 10 and $18 \mu \mathrm{m}$, indicating an amorphous lattice structure. In some Solar System comets (e.g. Hanner et al. 1994), and in the dust disk surrounding $\beta$ Pictoris (Knacke et al. 1993), evidence for a band near $11.3 \mu \mathrm{m}$ was found suggesting the presence of crystalline silicates in addition to amorphous ones.

Spectra of circumstellar dust shells taken with the Short and Long Wavelength Spectrometers (SWS, de Graauw et al. 1996; and LWS, Clegg et al. 1996) on board of the Infrared Space Observatory (ISO; Kessler et al. 1996), revealed the presence of many relatively narrow emission bands (e.g. Waters et al. 1996; Waelkens et al. 1996; Crovisier et al. 1997). A recent comprehensive study of ISO spectra of circumstellar dust by Molster et al. (1999) finds more than 50 (!) bands, mostly at wavelengths longward of $20 \mu \mathrm{m}$. Comparison with laboratory spectra showed that the majority of these bands is caused by crystalline silicates (hereafter XSILS), mostly by olivines $\left(\mathrm{Mg}_{2-2 x} \mathrm{Fe}_{2 x} \mathrm{SiO}_{4}\right)$ and pyroxenes $\left(\mathrm{Mg}_{1-x} \mathrm{Fe}_{x} \mathrm{SiO}_{3}\right)$ (Jäger et al. 1998). In addition, there may be $\mathrm{SiO}_{2}$ present (with peaks near 9 and $20 \mu \mathrm{m}$ ). A broad band near $65 \mu \mathrm{m}$, which blends with the $60 \mu \mathrm{m}$ crystalline $\mathrm{H}_{2} \mathrm{O}$ band, may be due to diopside $\left(\mathrm{CaMgSi}_{2} \mathrm{O}_{6}\right)$ (Koike et al., in preparation; Molster 2000). Crystalline silicates have up to now been found in the dusty outflows of evolved stars, in the disks surrounding young stars, and in Solar System comets. The abundance of XSILS compared to the amorphous grains shows a wide range, from a few per cent up to 75 per cent (Molster et al. 1999). The $\mathrm{Fe} / \mathrm{Mg}$ ratio of the olivines and pyroxenes can be 
measured since the peak of the emission bands shifts to longer wavelength with increasing $\mathrm{Fe} /(\mathrm{Fe}+\mathrm{Mg}$ ) ratio (Koike et al. 1993; Jäger et al. 1998). In all cases studied so far the $\mathrm{Fe} /(\mathrm{Fe}+\mathrm{Mg})$ ratio is less than about 5 per cent, and could be as low as 0 . This is in contrast with the $\mathrm{Fe} /(\mathrm{Fe}+\mathrm{Mg})$ ratio in amorphous silicates, which may be as high as 50 per cent. Since XSILS have many emission bands, it is possible to derive a colour temperature from band strength ratios. The XSIL temperatures are usually lower than those of the amorphous silicates. This is probably due to the low Fe content of XSILS, resulting in a poor absorptivity in the near-IR, where the contribution of $\mathrm{Fe}^{2+}$ dominates the absorption.

The temperature difference between XSILS and amorphous silicates strongly suggests that these are separate grain populations: a grain is either amorphous and Fe-rich, or it is crystalline and Mg-rich. This temperature difference may be the reason why XSILS so far have mostly been found in circumstellar dust shells with low $(\mathrm{T}<200 \mathrm{~K}$ ) colour temperature (Kemper et al. 2000). In such cool shells the contrast between XSILS and the warmer amorphous grains is smaller than in high temperature dust shells. Stars with high (low) mass loss have optically thick (thin) dust shells and low (high) colour temperature. The lack of emission bands of XSILS in the ISO spectra of stars with low mass loss rates therefore does not neccessarily imply that these objects do not produce XSILS, as was suggested by Waters et al. (1996). In evolved stars, the XSILS make out 10 to 15 per cent of the dust mass. There are also objects in which the XSIL abundance exceeds 30 per cent, and all of these stars have circumstellar disks (Sect. 2).

The presence of XSILS has also been established in the disks of some young pre-main-sequence stars (e.g. Malfait et al. 1998; Bouwman et al. 2000). The most spectacular case so far is HD 100546, whose ISO-SWS spectrum is dominated by forsterite $\left(\mathrm{Mg}_{2} \mathrm{SiO}_{4}\right)$ bands at 11.3, 16.5, 19.5, 23.5 and $33.5 \mu \mathrm{m}$, and shows a remarkable similarity to that observed in the Solar System comet Hale-Bopp (Crovisier et al. 1997). It is possible that in HD 100546 we are witnessing the perturbation of a large population of comets, resulting in the release of small grains consisting of forsterite.

Remarkably, evidence for the presence of XSILS in the interstellar medium (ISM) has so far been very scarce. Studies by Demyk et al. (1999) and by Vriend and Tielens (in preparation) have shown that the abundance of XSILS in lines of sight towards heavily embedded protostars and towards the galactic centre is less than about 2 per cent. Cesarsky et al. (2000) report the presence of XSILS in a star forming region, but Kemper et al. (in preparation) suggest an alternative identification for the emission bands. The apparent lack of interstellar XSILS opens the question of the life cycle of these grains: their low abundance in the ISM suggests that the release of XSILS into the ISM is much lower than the ISO observations of red giant stars suggest, or that the XSILS are destroyed in the ISM.

\section{The formation of crystalline silicates}

The formation of XSILS in circumstellar shells is not well understood. In the case of mass-losing evolved stars, silicates are formed from gas-solid nucleation near the stellar photosphere, and at temperatures close to the glass temperature 


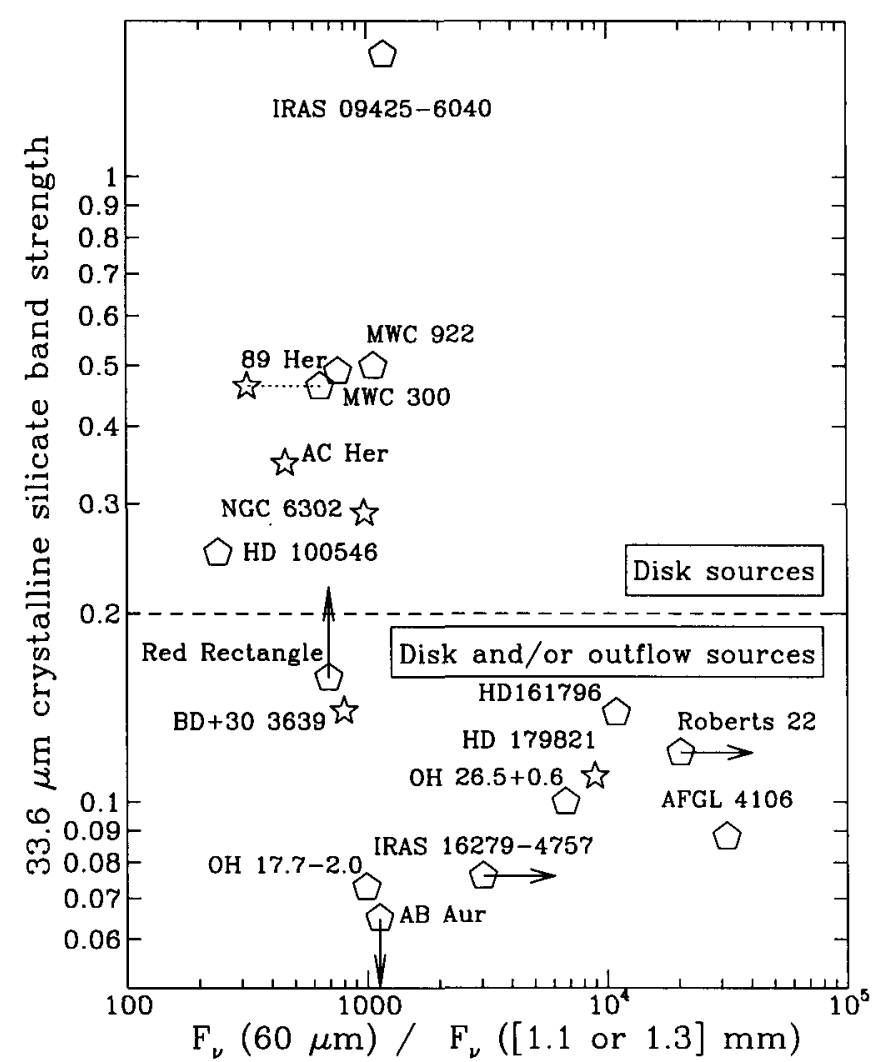

Figure 1. The correlation between the strength of the $33.5 \mu \mathrm{m}$ forsterite band is plotted against the ratio of fluxes at $60 \mu \mathrm{m}$ and 1.1 or $1.3 \mathrm{~mm}$, for a sample of evolved stars with cool dust shells (Molster et al. 1999). For completeness some young stars are also included. All sources with high abundance of XSILS have a disk-like geometry. The lack of sources in the upper right part of the diagram indicates that highly crystalline dust (as measured from the $33.5 \mu \mathrm{m}$ peak strength) correlates with the presence of large dust grains (as measured from the ratio of $60 \mu \mathrm{m}$ over millimeter flux). Conversely, coagulation does not imply crystallisation.

(about $1100 \mathrm{~K}$ ). Thermal annealing of particles in the condensation region may result in (partial) crystallisation of the grains (e.g. Gail \& Sedlmayr 1999). Kozasa \& Sogawa (1999) propose that $\mathrm{Al}_{2} \mathrm{O}_{3}$ grains form and obtain a mantle of crystalline silicates. This mechanism does not explain the difference in chemical composition of amorphous and crystalline silicates. Tielens et al. (1998) suggest that Mg-rich XSILS form at temperatures of about $1500 \mathrm{~K}$ and that most of these grains absorb $\mathrm{Fe}$ at temperatures below $1000 \mathrm{~K}$, destroying the lattice 
structure. A small fraction of the grains does not absorb Fe and remains $\mathrm{Mg}$ rich and crystalline.

The very high abundance of XSILS in some evolved stars with stationary, long-lived circumstellar disks is remarkable (Molster et al. 1999; see Fig. 1). These disks also show strong evidence for grain growth through coagulation. The disks have probably been formed during a phase of very high mass loss, possibly in a binary. However, AGB stars with on-going mass loss do not show such high abundance of XSILS. It is likely that the grains have crystallised in the disk, at temperatures that exclude thermal annealing as cause for the crystallisation. This low-temperature crystallisation may be due to spot heating of the grains resulting from electric discharges, or from bombardment with energetic particles.

\section{References}

Bouwman, J., de Koter, A., van den Ancker, M.E., et al. 2000, A\&A 360, 213

Cesarsky, D., et al. 2000, A\&A 358, 708

Clegg, P., Ade, P.A.R., Armand, C., et al. 1996, A\&A 315, L38

Crovisier, J., et al. 1997, Science 275, 1904

De Graauw, Th. et al. 1996, A\&A 315, L49

Demyk, K., Jones, A.P., et al. 1999, A\&A 349, 267

Gail, H.-P., Sedlmayr, E. 1999, A\&A 347, 594

Hanner, M.S., et al. 1994, Icarus 112, 490

Jäger, C., Molster, C., Dorschner, J., et al. 1998, A\&A 339, 904

Knacke, R.F., et al. 1993, ApJ 418, 440

Kemper, F., Waters, L.B.F.M., de Koter, A., et al. 2000, ESA-SP 456 (in press)

Kessler, M.F., et al. 1996 A\&A 315, L27

Koike, C., Shibai, H., Tuchiyama, A. 1993, MNRAS 264, 654

Kozasa, T., Sogawa, H. 1999, Asymptotic Giant Branch stars, eds. T. le Bertre, A. Lebre and C. Waelkens, page 239

Malfait, K., Waelkens, C., Waters, L.B.F.M., et al. 1998, A\&A 332, L25

Molster, F.J., et al. 1999, Nature 401, 563-565

Molster, F.J. 2000, thesis, University of Amsterdam

Tielens, A.G.G.M., et al. 1998, APSS 255, 415

Waelkens, C., et al. 1996, A\&A 315, L245

Waters, L.B.F.M., et al. 1996, A\&A 315, L361 